\section{Biochemistry panel dropped from UK quality review}

[LONDON] In a surprise - though widely welcomed - move, Britain's higher education funding councils have removed the biochemistry panel from the list of groups that will carry out its forthcoming Research Assessment Exercise (RAE).

The RAE is an initiative taken every few years to assess the quality of UK research, and is used by the funding councils to provide additional funds for more productive research departments. The next RAE will begin in 2001.

In the past, biochemistry has been assessed separately from the rest of the biological sciences, although this has been seen as illogical by many in both communities. For many years, senior figures had been unsuccessfully pressing for a merger of the panels. Biochemistry departments had been able to take part in the exercise under either panel, giving rise to doubts whether they were being judged on a comparable basis.

Another anomaly was forecast by the Biochemical Society when it began a campaign to have the two panels merged before the last exercise in 1996. It warned that keeping a separate biochemistry panel would lead to only a small number of the best departments choosing to be assessed separately from the rest of the biological sciences.

In line with this warning, in the 1996 exercise, only 17 departments went through the RAE under the biochemistry panel and a notably high proportion of these went on to be rated as 5 or $5^{*}$, the top categories in a seven-category rating scale.

According to some researchers, many of these departments appear to have engaged in strategic 'game playing'. By putting a small, high-level biochemistry department through the RAE separately from the rest of a large biological sciences department, it is easier to win top ratings - bringing the hope of receiving extra money.

As recently as last November, after sending a "very focused letter" on the subject, the Biochemical Society thought it had failed to convince the funding councils. The letter urged the councils to remove the panel.

The society has welcomed the change. Details emerged when the Higher Education Funding Council for England released a list of the chairs of unit of assessment panels two days before Christmas. Notably absent was any mention of a biochemistry panel.

"We conducted a fairly extensive consultation last year," says John Rogers, manager of the RAE. "The decision was taken in November to discontinue the biochemistry panel. We are responding to views received to improve the exercise."

NatashaLoder

\title{
French scientists offered time to set up companies
}

[PARIS] French researchers working for public research agencies and universities are to be offered up to six years sabbatical leave to set up their own companies. Scientists would retain their civil servant status during this period and have to choose at the end between a public or private sector career.

The widely expected move was announced last week by Claude Allègre, the minister of national education, research and technology, as part of a package of measures in a bill on "innovation and research" that will go before parliament later this year.

The provisions of the bill also include lifting a ban on scientists, as civil servants, holding shares or sitting on the boards of companies in which they have a direct interest. This has been a major obstacle to researchers aspiring to create their own companies, says Joseph Baexeras, deputy director of industrial development at the Centre National de la Recherche Scientifique (CNRS), France's basic research agency.

A key point of the proposed legislation is that researchers who set up companies would be encouraged to retain close links with their original laboratory, says Baexeras. He points to the absurdity that it is currently illegal for researchers to work for a company that has links with their home laboratory.

Whereas past government industrial policy initiatives have often centred on trying to improve links between public research and the private sector, the proposed law emphasizes the creation of companies as the major means of improving technology transfer and creating high-technology jobs.

Dominique Strauss-Kahn, the industry and finance minister, recently announced a multi-million-franc package of measures to stimulate innovation. These included FFr1 billion (US\$176 million) for new national networks of public- and private-sector laboratories in key technologies, and FFr600 million of public funds to boost venture capital (see Nature 393, 203; 1998).

The emphasis of the new measures is on identifying and developing promising projects to the stage where they would qualify for venture capital funding. To encourage this, the bill would free industrial development units within the research bodies from the administrative burdens associated with being part of a public body.

Such units would instead be run along the lines of a commercial company, with greater flexibility, for example, in decisions on hiring staff and spending budgets. The creation of joint subsidiaries between research bodies and companies currently requires approval signed by several ministers, but in future such approval would be tacit.

The CNRS intends to create an 'incubator' unit for start-up companies that would provide training in business and finance for scientists. Promising projects would be funded by a series of 'seed money' funds.

The state budget for research this year includes a FFr200 million seed money fund which will be distributed following a call for proposals in the spring. Allègre also promised to launch a competition this year with FFr100 million to be awarded to the most promising start-ups, in particular in information technology and biology.

The Atomic Energy Commission (CEA) announced last week that it would create a seed money fund of FFr160 million for microelectronics. Plans are also afoot to create a fund for biotechnology. Declan Butler

\section{US genome researcher strikes it rich}

[WASHINGTON] For genome researcher and baseball fan Philip Ozersky of Washington University, St Louis, Mark McGwire's record breaking 70 home runs in the last baseball season was not only an event of semireligious status, but also highly lucrative.

Ozersky, who attended the game with colleagues from the university, was able to catch the ball, which was sold at auction last week to an anonymous bidder for just over $\$ 3$ million -23 times the previous record price for a baseball.

Ozersky, who attended the auction wearing a tie decorated with a double helix, and says that he will give some of the money to charity, has no plans to give up his work on human chromosomes 7 and 22.

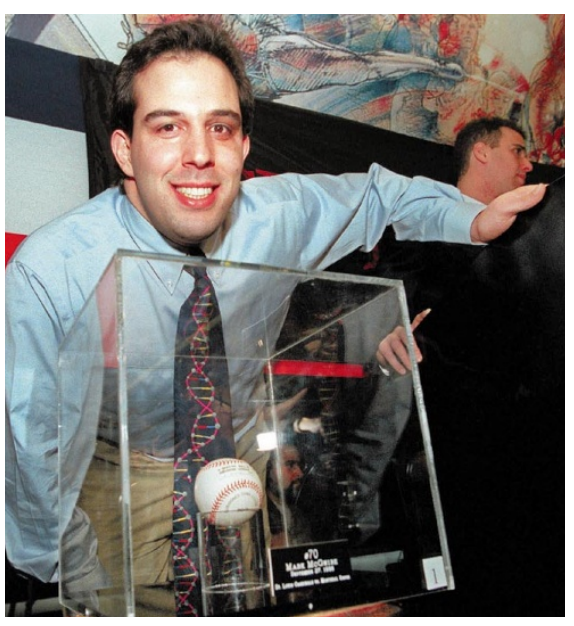

